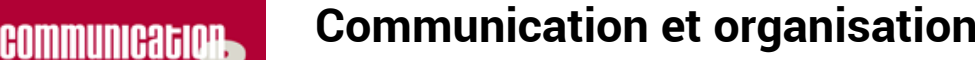

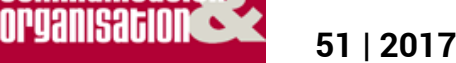

Les nouvelles cultures de l'information dans les organisations

\section{Textes et échanges dans une organisation}

Prescrire comme travail d'organisation : le cas d'une administration d'État et de ses services (la Protection Judiciaire de la Jeunesse en France)

Texts and exchanges within organizations. Prescribing as organizational task: the case of a State Service and its departments (the French "Protection Judiciaire de la Jeunesse" - Youth Judiciary Protection)

\section{Pierre Delcambre}

\section{(2) OpenEdition}

1 Journals

Édition électronique

URL : http://journals.openedition.org/communicationorganisation/5567

DOI : 10.4000/communicationorganisation. 5567

ISBN : 979-10-300-0146-4

ISSN : $1775-3546$

Éditeur

Presses universitaires de Bordeaux

Édition imprimée

Date de publication : 1 juin 2017

Pagination : 123-140

ISBN : 979-10-300-0162-4

ISSN : $1168-5549$

\section{Référence électronique}

Pierre Delcambre, «Textes et échanges dans une organisation», Communication et organisation [En ligne], 51 | 2017, mis en ligne le 01 juin 2020, consulté le 02 janvier 2021. URL : http:// journals.openedition.org/communicationorganisation/5567 ; DOI : https://doi.org/10.4000/ communicationorganisation. 5567 


\section{Textes et échanges dans une organisation. \\ Prescrire comme travail d'organisation: le cas d'une administration d'État et de ses services (la Protection Judiciaire de la Jeunesse en France)}

\section{Pierre Delcombre ${ }^{1}$}

Mon titre annonce mon point de vue : faire entrer en discussion des approches différentes ; (1) celle de l'organizing (« texte» - « conversation») ; (2) de l'activité managée, outillée, mais aussi opérée grâce, certes, aux opérationnels, mais aussi au «travail d'organisation » de l'ensemble des salariés, dès lors que des dispositifs impersonnels ne réduisent leur agentivité à néant ; (3) et enfin des communications en contexte de travail.

Dès lors, la question est : sur quels textes, sur quels échanges peuvent prendre appui les méthodologies des uns et des autres ? Je m'appuierai sur un récent travail ( Prescrire, une étude de la prescription dans l'univers d'une administration régalienne - ministère de la justice en France- : la Protection judiciaire de la jeunesse chargée des mesures concernant les mineurs " au pénal ») pour faire un point sur ce que nos méthodologies d'étude des communications de travail et des cadres d'échanges peuvent inciter à développer comme théorie de " l'écrit » et de "l'oral », des textes et des échanges au travail. Une discussion qui a déjà une histoire...

Mon objectif, ce jour, est de participer aux discussions déjà en route 2 . Dans mon titre, je pose 3 termes : " travail d'organisation » (et non pas processus organisant), « textes » (au pluriel) et « échanges » (et non pas conversation) Signaler ces décalages me permet de dire d'abord que je ne vise pas une " critique interne " des positions de la $\mathrm{CCO}$, elle sait le faire fort bien toute seule et sait dire que "le monde organisationnel est un monde pluriel, dont le mode d'existence ne semble pas se réduire à sa seule actualisation communicationnelle " (Cooren, 2010:24)! Je cherche aussi à assumer un décalage théorique que je décrirais ainsi : je ne travaille pas directement à une sociologie des organisations - ni une théorie du management, ni une

1 Professeur Emérite, Geriico, Université Lille3 ; pierre.delcambre@univ-lille3.fr

2 Ce travail a été présenté et discuté lors d'un séminaire commun à Gériico-Lille3 et au Lasco-UCL, en présence de F.Cooren et F.Lambotte fin juin 2016. Merci à Anne Mayère pour sa relecture critique. Certaines notes et références sont redevables à François Cooren. Merci à lui, aussi. 
théorie de l'organizing - ou sur les "formes organisationnelles ", mais bien sûr les "formes communicationnelles ", en usage au cours de l'activité et du travail (Delcambre 2016b), des formes qui peuvent être comprises en combinant une socio-économie des formes et des conditions des échanges et une anthropologie des communications qui ont lieu « dans les organisations ».

Mais l'usage de ces mots (texte, conversation, échanges) signale aussi ma " cousinerie » avec les travaux Montréalais. Le chercheur que je suis, qui n'a jamais quitté les terres de "Langage et Travail ", ne pouvait que suivre avec intérêt les tentatives pour prendre en compte des phénomènes langagiers comme contribuant aux transformations des organisations ou à la construction même de l'organisation et ce même si les travaux que j'ai menés sur les communications au travail m'ont mené moins à explorer l'organizing qu'à comprendre les opérations sociales ${ }^{3}$ que mènent les gens au travail avec leurs collègues, leurs partenaires, leur hiérarchie.

Je travaillerai en trois temps. Je commencerai, dans un premier temps, par poser les différences de conditions d'enquête : quelle situation organisationnelle étudie-t-on si l'on traite des flux communicationnels sur des durées un peu longues, quels textes sont "stratégiques " pour l'organisation, "pertinents " pour le chercheur et comment ajuster la méthode d'enquête ? Dans un second temps j'exploiterai plus précisément la situation que j'ai pu étudier, concernant une dynamique de prescription (une opération sociale « managée » : prescrire). Quelle performativité l'organisation assure-t-elle au texte ? Enfin, dans un troisième temps, je viserai à indiquer les dialogues et complémentarités que les trois approches prises en compte ici donnent à penser.

\section{Méthodologies pour étudier les transformations de l'activité dans une organisation "en altération ${ }^{4}$ de forme organisationnelle"}

Le présent texte s'appuie sur la lecture de deux articles de nos collègues belges (Jojczyk, Lambotte, Mnasri et Wathelet 2016, dorénavant JLMW et Lambotte et Wattelet, à paraître, 2016). Ils travaillent les questions théoriques adressées à la CCO par un biais méthodologique en inventant des méthodologies congruentes avec leurs objectifs théoriques ${ }^{5}$ : c'est une entrée essentielle pour la discussion. Lambotte \& Wathelet sont ainsi d'autant plus sensibles aux « moments " (que sont les moments-texte et les moments-

\footnotetext{
3 J'utilise ici parfois « opérations sociales » que je cherche à distinguer de «situations de communication ». Dans le cadre de cet article, je ne peux m’en justifier longuement. Disons pour l'instant qu'une opération sociale est une action et un échange identifié et désigné par ceux qui y participent. Cet échange suppose une action combinée de celui qui mène l'action et de celui qui accepte de mettre en œuvre ce que l'autre lui demande. Une relation asymétrique et complémentaire le plus souvent, donc.

4 Je reprends le terme d'altération aux travaux de Christian Le Moënne (Le Moënne 2015, Le Moënne \& Gallot 2015).

5 Je comprends ces objectifs comme une analyse plus centrée sur le management (ici le management du changement) que sur l'organisation. Dès lors observer comment des moments de communication contribuent à l'organizing est méthodologiquement stratégique chez eux. Lobservation des échanges leur permet de comprendre un « management en acte », avec ses tentatives, ses réussites et ses échecs.
} 
conversation) que le «moment historique » qui fait la situation de leur propre enquête est une période d'un changement voulu, appuyé sur des consultants et « managé ».

De fait, les terrains d'enquête (leur durée, mais aussi le moment où se tient l'enquête dans l'histoire du management d'une organisation) ont le plus souvent des particularités qu'il faut expliciter pour que la discussion sur communication et organizing puisse être fructueuse.

De notre côté, l'enquête collective portée par Patrice De la Broise et Céline Matuszak dans des établissements de la protection judiciaire de la jeunesse (Delcambre et Matuzsak 2016) concernait une durée longue de réaménagements organisationnels managés, propres à la « réadministration » de cette direction du Ministère de la Justice (Chéronnet 2016) et au renforcement d'aspects répressifs de la politique de prévention de la délinquance, en France. Progressivement nous nous sommes rendu compte que la période à considérer nétait pas celle de notre enquête sur le terrain (2009-2011), ni celle où la Direction de la PJJ posait un « Projet Stratégique National - PSN » (20082011), mais plutôt une période démarrant dans les années 2002-2003 et non clôturée à la fin de l'enquête. Une période de multiplication, voire d'accélération normative (Heller, 2016). Ainsi, le périmètre temporel peut certes être défini par le chercheur en fonction de ses préoccupations et questionnements, mais il est nécessairement repensé sur le terrain ${ }^{6}$.

Nous nous focalisions sur les "effets normatifs " de toute une série de normes et prescriptions managériales sur les pratiques des " opérationnels » (ici essentiellement des Educateurs de la PJJ, et plus particulièrement sur une partie de leur activité dite stratégique dans le PSN : écrire au magistrat). La conséquence est une méthodologie de recueil, d'enquête et d'observations de terrain au niveau opérationnel des Unités et Services de la PJJ, niveaux hiérarchiques les plus « bas ».

Enfin, cherchant à étudier de plus près la prescription et comment celleci prend effet dans un moment d'accélération normative et prescriptive, le repérage local de textes, d'outils, de machines, de dispositifs contribuant à la prescription m'a amené, dans l'enquête que j'ai personnellement menée, à « remonter » à partir du terrain vers d'autres niveaux de la chaîne hiérarchique. "Remonter » est le mot du chercheur engageant des méthodes d'enquête ; mais cela veut dire que l'organisation communicationnelle s'étudie, elle aussi, que les formes et formats de nombreux échanges sont prévus, envisagés, agis par tous les niveaux qui contribuent au « travail d'organisation ».

A priori, on pouvait penser que s'il y a des documents textes qui peuvent fonctionner comme références stabilisatrices pour l'organisation, ce seraient bien les textes dits stratégiques. Une autre catégorie de «texte »/textes qui pourraient contribuer à «stabiliser l'organisation » peuvent être les textes

6 Je remercie A. Mayère pour la formulation explicite de cette idée. 
prescriptifs. Ils stabilisent divers compromis établis dans des espaces sociaux très divers. Ces textes ayant une visée prescriptive se développent notamment dans des chaînes textuelles-documentaires (on peut considérer la chaine loiscirculaires-note d'application comme tels). De tels textes (notes, circulaires, mais aussi "référentiels ") sont repérables, bien sûr, aussi dans le " monde " organisationnel que nous étudiions, la PJJ.

Comment « fonctionnent-ils »? D'où vient l'efficacité - conçue comme une mise en œuvre "observante "- de la prescription ? Du texte agité comme référence par des hiérarques puissants ? Des attitudes " observantes » de salariés contributeurs? Du contrôle de l'observance?

Qu'arrive-t-il quand une organisation multiplie les prescriptions et les textes prescriptifs ? Qu'est-ce qui fait que ce «trop » (un ressenti repérable à bien des niveaux hiérarchiques, et surtout aux niveaux de l'organisation qui n'ont pas à « instruire le prescrire " mais à " opérationnaliser localement les missions ») ne tombe pas de soi-même comme "irréel " mais que certains de ces textes prescriptifs donnent lieu à une mise en œuvre ? Existe-t-il des « textes prégnants »?

Ce sont ces questions qui m'animaient.

Un mot pour finir ce moment de précisions quant aux cadrages de la recherche sur laquelle je m'appuie aujourd'hui : mon orientation théorique combine l'observation des communications de travail et celle de l'activité. Dès lors je suis attentif à la contribution des salariés au « travail d'organisation ». Si l'on considère que l'activité est avant tout l'inverse d'une application ou d'une exécution de normes, mais bien une production de normes dans une situation sociale multidéterminée qu'elle modifie en retour... alors, dit M-A.Dujarier, " toute activité est doublée de ce que nous appelons à la suite de Gilbert de Terssac un travail d'organisation (...) Il s'agit de délibérations utiles pour bâtir, en dépit des tensions qui la trament, des finalités, des procédés et des justifications sociales à l'activité. » (Dujarier 2015 : 31).

Je dois aussi préciser un point théorique qui oriente ma recherche et mes inventions de méthodes : ce point théorique, dont je ne mesure pas toutes les implications, est que je considère que la prescription " manque à prescrire » (la prescription passe par une explicitation le plus souvent textuelle de ce qu'il y a lieu de faire, à la différence de la norme, qui consacre ce qu'il est «bon de faire ", dans le registre des valeurs, la norme sociale ou professionnelle ne devant souvent pas être explicitée pour faire son effet $\left.{ }^{8}\right)$. C'est parce que la prescription est à la fois explicite, textuelle et qu'elle " manque à prescrire ",

7 Cette dernière formule m'est suggérée par A. Mayère, qui s'appuie sur les « indicateurs prégnants » de V. Boussard et renvoie à de Gaulejac pour estimer que ce serait une caractéristique du capitalisme contemporain que d'édicter des normes proliférantes et paradoxales. Dès lors, c'est à l'enquête de repérer les « textes prescriptifs prégnants » et à comprendre comment ils ne s'irréalisent pas par obsolescence, dé-motivation.

8 Les chercheurs qui visent à travailler sur l'évolution des normes sociales et/ou des normes professionnelles ont besoin de prendre en compte des durées encore beaucoup plus longues. Pour la PJJ, voir Sallée (2010) et Jurmand (2009). 
notamment parce qu'elle ne réussit pas à dépasser les contradictions et tensions propres aux orientations normatives concurrentes pour l'activité ordinaire des opérationnels, que la prescription est prise dans un " travail d'organisation ", à tous les étages. Ici, dans une organisation qui se veut institution, elle est à chaque fois un moment de travail de « renormalisation de la prescription ». Et pourtant, il y a encore du travail « local » à faire par les services et les équipes.

\section{Prescrire à la PJJ: un travail d'organisation fait de textes certes, mais de quoi d'autre?}

«Les rapports sociaux sont médiés par des choses et des dispositifs qui s'interposent entre prescripteurs et travailleurs» note M.-A. Dujarier (Dujarier 2015 : 69) Au cours de cette enquête il m'a été possible de mettre en lumière au moins quatre processus ou dispositifs assurant la mise en œuvre de la prescription. Certes cette étude amène à décrire des modes d'exercice de pouvoirs dans l'organisation, mais aussi des modes de contribution des salariés, dans toute la chaîne organisationnelle. Ces dispositifs mettent en jeu plusieurs niveaux hiérarchiques, non pas forcément en " cascade ", mais dans des jeux de "réinterprétations " ${ }^{9}$. Ces différents dispositifs, tous producteurs de textes, d'échanges, de moments de réunion, semblent souvent se référer les uns aux autres. J'utiliserai dans les paragraphes qui suivent le terme d'" outil de la prescription » dans la mesure où ils sont mis en ouvre comme un «équipement de la prescription », jouant à des moments différents pour parfois instruire, parfois contrôler, parfois légitimer. Ils sont construits et mis en œuvre par des services souvent différents, ne sont pas forcément " articulés »- pour reprendre Strauss, si souvent pertinent pour une analyse de l'activité « conjointe », dans son terrain habituel, « le travail de santé »mais concourent à la réussite des actions prescrites.

\section{La chaîne de prescription et le «travail d'organisation»}

D'une part, il y a bien la chaine des textes prescriptifs, mais qu'il faut concevoir comme matière textuelle du travail d'organisation. Ici, il s'agit d'une loi, la loi 2002-2 "rénovant l'action sociale et médico-sociale », connue comme loi définissant les «droits des usagers » dans ce secteur, loi appuyée sur une série d'outils dont nombre sont des textes-documents. Cette loi comporte 87 articles. Une note d'application propre à la PJJ de mars 2007, publiée par le Directeur de la PJJ, administration du Ministère de la Justice, elle, est donc publiée cinq ans après la loi, après une procédure de travail d'un groupe de personnes nommées dans le document même. Ce document comporte plus de soixante pages, associe des annexes de type «formulaires " et " grilles

9 C'est bien dans le processus de réinterprétation que l'on retrouvera la " convocation » d'une référence, la citation ou la ventriloquie de Fr. Cooren repère dans les interactions. 
d'écriture » qui présentent un « prêt à l'emploi » ajusté à la PJJ pour aider à la mise en ouvre de la loi.

Il n'en reste pas moins qu'un " travail local d'organisation » reste à faire, par des discussions : un texte, fût-il réputé coercitif-normatif-réglementaire, ne vaut que si un travail d'organisation l'actualise. Ici, nous ne sommes pas dans un processus organisé par " procéduralisation » qui effacerait l'agentivité - donc la contribution et le travail - des acteurs locaux et enrégimenterait les opérationnels dans l'actant « logiciel » ou ERP qui « ferait faire » (Bazet \& Mayère 2016). Lordre du jour même du travail local d'organisation se révèle stratégique. C'est ce «travail d'organisation » qui, au niveau local, est un travail de "renormalisation de la prescription " selon Terssac (Terssac 2002) ou Dujarier (Dujarier 2006 et 2015) : la direction locale, entourée de l'équipe, discute des modalités de la mise en œuvre : discussions, controverses, propositions concurrentes, décision collégiale ou imposée. Ce processus suppose donc un "relais de prescription " (Denis 2007) : la hiérarchie de proximité.

\section{Contrôle de conformité et audit interne}

Dès lors, il y a bien un second « outil de la prescription » : le contrôle des managers de proximité et de leur action, par l'audit (il faut noter que la mise en place de services PJJ d'audit est très récente et ne concerne les services directement administrés que depuis 2011). Un audit est aussi fait d'entretiens, de textes, de discussions. Les auditeurs pour faire leur travail - ici un audit de conformité, non de qualité - s'appuient sur une liste de 30 items de textes de référence, classés par ordre de force normative : du Code de l'action sociale et des familles, en passant par des articles de l'ordonnance de 45, des lois, décrets, circulaires, puis des notes, mais aussi le référentiel « Répertoire des mesures confiées... » et le Projet Stratégique National.

Le travail même d'audit est cadré par des circulaires : une liste précise des " moments d'échanges» et des textes produits, ainsi que leur sens dans le phasage du processus d'audit. Cela donne un " planning de l'audit » (un document d'une page organisant les rendez-vous locaux sur quatre jours); puis un pré-rapport d'audit (trois mois après), transmis par courrier recommandé, " pour la période (réputée) contradictoire ", à la Directrice du service. Un mois après, le pré-rapport est réputé « définitif ». Ce rapport d'audit territorial comporte 46 pages avec conclusion « Points en exergue, axes d'amélioration et rappel des préconisations » pour un total de 20 préconisations (pages 16 à 40). Les Auditeurs préparent alors un Plan d'action discuté et stabilisé lors d'une réunion d'une matinée, trois mois plus tard, de « restitution de l'audit » à la Direction Territoriale concernée. Ce plan fait six pages, est présenté en trois colonnes (Constats des Auditeurs ; Rappel de la préconisation ; Échéance) ; il suit l'ordre des préconisations du rapport. 
Il est difficile pour moi d'utiliser le terme de « conversation ", comme mot d'usage courant et comme concept, notamment pour rendre compte des propos tenus dans cette dernière réunion : il ne s'agit guère d'une " refluidification » de l'organisation, mais bien d'engagements de transformation de points précis de l'organisation locale, laquelle reste à faire.

\section{Un outil informatique permettant de consacrer des «indicateurs» de gestion}

Un troisième outil apparaît dès lors que l'on s'intéresse à l'activité locale : un logiciel (ici appelé GAME : Gestion de l'Activité et des Mesures Educatives) qui intègre dans sa conception des aspects prescrits de l'activité (répartition des mesures, nombre de mesures par professionnel, durée entre la notification de la mesure et son attribution à un « référent $)^{10}$. Une part de la prescription donne ainsi lieu à des indicateurs et à l'implémentation par les contributeurs des niveaux opérationnels (Responsable d'unité assisté de sa secrétaire): c'est une part notable de l'agentivité de certains aspects de la prescription. Ces indicateurs sont calculés par la machine, sont évocables... et évoqués notamment lors de la restitution de l'audit, par le Responsable Territorial de la gestion des services.

Le texte-document «référentiel» (des mesures et des missions confiées aux services de la direction de la protection judiciaire de la jeunesse)

J'ai pu étudier cet autre outil, qui nous était collectivement apparu, en démarrage de recherche, comme "prescriptif ", et susceptible de normaliser les pratiques par «standardisation » : un référentie ${ }^{11}$ définissant la dynamique de travail propre à chacune des mesures en usage au moment de cette publication (soit septembre 2005). Ici, l'enquête a fait ressortir d'abord que le référentiel publié, devenant obsolète, nétait pas ré-actualisé. Elle a montré ensuite que les professionnels disaient « connaitre le référentiel »; or les pratiques observées donnaient à voir que les professionnels réduisaient considérablement la diversité prescrite dans l'exercice de mesures différentes. On peut faire l'hypothèse que ce texte-document de 165 pages a été un effort de la hiérarchie PJJ pour stabiliser des pratiques, voire les homogénéiser sur tout le territoire et pour la variété des services ayant à mettre en œuvre des mesures.

Si l'on analyse " prescrire " comme une " opération sociale " mettant en relation deux partenaires de l'échange et de l'action (le prescripteur, sollicité

10 Ici on peut faire référence aux études qui montrent le rôle des outils de gestion dans la « conformation » et la prescription : ils contribuent à formater l'action (Moisdon 1997).

11 Référentiel des mesures et des missions confiées aux services de la Direction de la Protection judiciaire de la jeunesse. Publication de la Direction de la protection judiciaire de la jeunesse, sous-direction des méthodes éducatives et des affaires judiciaires, Édition Septembre 2005, 165 pages. 
ou non ; à qui il est prescrit, sollicitant ou non), il est important de noter que la prescription suppose que le prescripteur connaisse quelque peu son partenaire pour que la prescription soit à la fois « ajustée » et susceptible d'être opérationnalisée (par une observance); dès lors le « référentiel » montre bien la connaissance construite par l'expert-prescripteur de l'activité des gens qu'il espère soumettre à l'observance (Delcambre 2016c).

Dans la perspective de la discussion d'aujourd'hui, je note que ce document, répondant à un effort ponctuel, n'a guère fonctionné comme "stabilisateur ", non que des discussions ultérieures l'aient « remanié » sans lui laisser le temps d'opérer une stabilisation (standardisation, normalisation), mais parce que comme objet, sa matérialité le rendait très vite obsolète. Lédition papier en a fait un document de référence connu, mais sans qu'on sache où il se trouve. On peut aussi se demander, pour ce document, quels agents pouvaient bien être les « relais de prescription ». Un référentiel nest pas « en soi » un instrument puissant de normalisation des pratiques décrites-prescrites dans le texte.

Ainsi l'organisation dispose de plusieurs « outils » contribuant à la performativité de certaines prescriptions existant sous forme textuelle.Mais ces outils sont plus ou moins « puissants » et supposent un engagement contributif et des managers de proximité, les plus contrôlés, et des équipes, des salariés, eux-aussi. Dès lors nombreuses sont les dynamiques communicationnelles de dispositifs personnels ou impersonnels.

\section{Discussion : texte/conversation et l'usage des "texte-textes- documents» et de "conversation-situations de communication- échanges-interactions"}

Les éléments que je vais pointer ne sont pas ignorés de la réflexion $\mathrm{CCO}$ : il suffit de lire l'introduction que François Cooren fait de «Comment les textes écrivent l'organisation. Figures, Ventriloquie et Incarnation » (Études de communication $\left.\mathrm{n}^{\circ} 34,2010: 23-24\right)$ pour s'en rendre compte. Reprendre ou poursuivre des critiques n'a de sens pour moi que de confronter plus précisément des approches théoriques différentes.

Il me semble qu'il existe plusieurs interprétations du jeu « texte/ conversation ». Je ne vise pas ici une analyse exhaustive qu'un état de l'art pourrait apporter. La formule que je retiens comme précisant ce qu'il en est du jeu " texte/conversation (de sa dynamique ou de son cycle) est la suivante, issue de la première période CCO : «l'organisation se cristallise (devient texte) par les conversations et l'organisation est rejouée et remodelée (devient conversation) par les interactions " (c'est en quelque sorte la version Taylor \& Van Every 2000, 2011, 2014). Dès lors, le primat des interactions comme contributives/constitutives est clair, mais ces interactions contribuent à des conversations, et certaines se cristallisent en texte et sont rejouables en conversation. Dès lors encore, pour certains analystes, le texte " demeure », la conversation est " événementielle », en tout cas, les textes ont une existence 
aussi hors de l'interaction. Parfois on peut lire que le texte "sanctionne la fin d'une conversation » (JLMW p. 13) ou que le texte est «stabilisé » (des textes ont besoin d'être stabilisés comme texte, donc). Pour ma part, j’avais tendance à interpréter de la manière suivante : « le texte stabilise l'organisation, la conversation la "refluidifie" ", réinterprétation de ma part, certainement marquée par la sociologie institutionnaliste (conversation instituante vs texte institué $)^{12}$.

Ces différentes lectures me semblent recouvrir deux orientations théoriques différentes qui ne me semblent pas de la même nature :

- Pour la première : le texte contribue à l’organizing en stabilisant un état d'organisation, un état qui peut " représenter » l'organisation, et on pourrait même considérer l'organisation comme texte.

- Pour la seconde, lorganisation, comme lieu d'interactions à la fois stabilise des textes et les rejoue dans les échanges ordinaires (soit pour les confirmer soit pour les modifier). On reste alors centré sur les dynamiques d'interaction et on pose une préférence théorique pour une méthode d'observation d'une organisation impermanente sans jamais de «clôture du sens ».

Et j'ai bien lu dans Cooren 2010 déjà cité qu'il définit un jeu entre texte et interactions. Je note, pour ma part, que l'évolution de Cooren passe par un évitement du terme de conversation : "Selon cette perspective, l'agentivité des textes (qu'ils soient écrits ou oraux ${ }^{13}$ ) doit donc être comprise comme la médiation par laquelle une pléthore de figures à ontologie variable s'expriment et s'incarnent dans l'interaction "... et que, dans le même article il propose d'étudier " comment les textes (je note le pluriel, remarque P.D) écrivent l'organisation ».

Revenons, dès lors, au texte et à la conversation.

\section{Texte vs textes; texte vs document}

Les collègues du LASCO déjà cités (JLMW) disent que l'observation propre aux recherches $\mathrm{CCO}$ a surtout porté sur les interactions. Si le phénomène était avéré, j'ai tendance à penser que c'est notamment parce que le texte semblait avoir une " évidence ». Triple évidence : matérielle ; documentaire, et donc conceptuelle. Cette triple évidence a permis de mettre les bouchées doubles sur la manière dont les conversations contribuaient à déstabiliser l'organisation instituée par un texte antérieur et comment elles « invoquaient» le texte.

Or, ces trois « évidences » n'en sont guère.

12 Fr.Cooren me signale que cette lecture est en phase avec la version CCO de Taylor et Van Every, dans la suite de Taylor et al, 1996. Son analyse est que, dans cette première construction de la CCO, l'approche théorique s'accroche à l'herméneutique de Ricœur 1986.

13 Cette formule est, elle aussi, notable. Elle me semble correspondre à une évolution vers une analyse de «l'écriture » du texte (traces, marques, texte). Mais, dans le même temps, elle permet de décrire des « conversations numériques » comme forme textuelle orale d'interactions ! 
Évidence matérielle et documentaire : sauf à donner au singulier « texte » une définition spécifique (le texte devenant un autre mot pour « organisation » dans l'interprétation de nos collègues belges, que ne soutiendrait pas la $\mathrm{CCO}^{14}$ ), le terme de texte avait l'avantage de stipuler une matérialité, et de permettre l'appui méthodologique, au besoin, sur la " génétique des textes ».Je garde pour ma part une définition du et des textes empreinte de matérialité : il a ou non des zones de texte déjà écrites, des espaces d'inscription à venir, une organisation formelle, un ou des supports, etc ${ }^{15}$. D'autre part, le texte n'est pas une simple addition d'énoncés ni l'émergence ou le ré-usage de motifs discursifs empruntés. Cette définition, à mon sens, devrait interdire de confondre texte et discours, non que les techniques d'analyse de discours ne soient pas pertinentes dans certains cas, mais étudier des discours me semble relever d'un autre univers théorique (voir la revue Langages ainsi que Gardin 1976).

Lévidence matérielle perd de sa force sous le coup de plusieurs phénomènes, dès lors que l'on s'intéresse aux textes dans leur pluralité. D'une part, bien des textes sont " oubliés ", inopérants, obsolètes. Seuls certains textes sont « invoqués ». D'autre part, il arrive souvent qu'ils n'aient pas de localisation matérielle - donc documentaire - connue des usagers : ils sont dans le souvenir de celui qui invoque, et, pour les textes prescriptifs, ils sont postulés par l'existence, elle concrète, des « relais de prescription» (Denis 2007). Enfin, si la question de l'adressage des textes (et de leur envoi effectif, de leur transmission adressée ou " par arrosage ») est stratégique dès la production d'un texte, pour les éditeurs-producteurs, l'intention momentanée d'un acteur et ou d'un collectif d'acteurs ne fait pas la circulation et la réinterprétation du texte.

C'est pourquoi la version Cooren 2016 a l'avantage d'accepter le principe de réinterprétation comme constitutif «du» texte. Dans cette perspective théorique, il est sage de traiter des réinterprétations. Reste à savoir comment... ${ }^{16}$

14 C'est ce à quoi amène chez JLMW la lecture de Cooren 2016 : « texte » est synonyme d'organisation... et pour prendre en compte les emboitements organisationnels, il faudrait même dire, dans leur perspective, "grand texte " pour les organisations plus globales productrices de règles, affectées par des compromis de normes, faisant « cadre " pour telle ou telle organisation locale. Ici, le danger de métaphoriser non seulement le terme de texte, mais aussi la performativité. Car, comment une organisation «performe »-t-elle ? Comme un texte ? Certes non! Et la question du pouvoir de faire dans l'organisation (agentivité) n'est pas celle du pouvoir des textes (dispositif d'efficience, voir Delcambre 2016a), et ne renvoie pas si simplement à la performativité de l'écrit traité par B. Fraenkel (2006) et encore moins celle de la performativité des écrits organisationnels sur une durée clôturable par la production d'interprétations et/ou de nouveaux textes.

15 Cela m’amène à préférer dire, en m'éloignant de Cooren ou de Vasquez, que la présence d'une « référence à un texte " dans une interaction est une évocation ou une invocation " discursive ", à la différence du geste qui met sous les yeux de l'autre le document-texte sous une de ses versions autorisées.

16 Accessibles méthodologiquement par la réitération de "la marque "? Mais comment la perspective Déridéenne concernant l'écriture amène-t-elle à étudier les textes? Dans leur épaisseur génétique ? Dans leur intertextualité faite de références? Dans leur dispersion faite de fragments donnant à penser des palimpsestes pour l'archéologue, et du bazar d'auteurités organisationnelles pour les supposés lecteurs, fragments devenus " immatériels » voire " immémoriels »? Une étude plus précise de Cooren 2013, que je n’ai pas faite, pourrait nous faire avancer. 
Évidence conceptuelle : quel texte, parmi les textes produits dans une organisation (dont on aurait défini les limites d'autorité sur des agents, sur des sous-traitants, des "partenaires ») peut être caractérisé comme cristallisant (stabilisant ?) un état d'organisation et ce sur une période définissable d'organisation. Pour ma part j'ai tendance à poser que ce n'est pas la nature d'un texte qui en fait un élément stabilisateur de «l'organisation ». Des dispositifs sont mis en place (organisés) pour que le texte "vaille » comme stabilisation, c'est ce que la partie précédente a voulu montrer.

Mais, dans certains cas (par exemple avec le PSN PJJ déjà évoqué), c'est au contraire le passage par la médiatisation externe et ses relais internes - cas classique du changement des administrations publiques, voir aussi le cas de La Poste, en France, années 1985- qui donnent à un document-texte une valeur qui permet qu'un texte cristallise l'organisation (moment d'organizing).

On noubliera pas, si on s'intéresse aux textes, que des luttes existent qui invalident des textes, leur faisant perdre momentanément ou durablement leur valeur pratique, leur « agentivité ».

Une contribution dans des moments d'échanges dans une organisation estce une «conversation»?

Conversation a été ici à mon sens un marqueur de référence : Sacks, Cicourel et Garfinkel (voir notamment Bachmann, Lindenfeld et Simonin, 1981 : 141-161), cherchant à définir le travail conversationnel de chaque partenaire de l'échange, et non pas du partenaire cadrant et définissant la nature du cadre de l'interaction. Une sociolinguistique ? Pas exactement... mais un vrai intérêt pour les gens, pas tout à fait les "participants " d'une ethnographie de la communication (Hymes, voir Bachmann, Lindenfeld et Simonin, 1981 : 72-76), plutôt une interprétation plus Greimassienne qui a conduit à penser tous les « actants » de l'organisation, la chose est connue. Très vite si l'analyse pouvait être " conversationnelle ", elle a pris en compte, avec les interactions, des «cadres", pas que des contextes (De Fornel, Joseph...). Mais « conversation » est aussi devenu une catégorie appuyée sur une psychosociologie de la communication : se développe alors une analyse différenciée des «situations de communication ». "Conversation » est alors différenciée du débat, de la dispute, de la consultation, etc.: elle est comprise comme symétrique et non pas complémentaire, ce qui impliquerait que des acteurs sociaux (ici en contexte de travail) acceptent de mettre entre parenthèses leurs rôles sociaux - plus ordinairement complémentaires - pour accepter la symétrie lors de l'échange, phénomène supposant, je pense, engagement de familiarité ou engagement de citoyenneté ! Cette symétrie est protégée parfois par une gestion de l'espace communicationnel, rarement dédié, sauf dans les "salons " de la fin du $18^{\text {e }}$ et du $19^{\text {e }}$, et surtout par des normes de civilité dont l'histoire remonte aux cours princières italiennes (1527, Castiglione Il 
libro del cortegiano, notamment)..., plus que de démocratie formelle (c'est le « débat » qui renvoie à ces normes-là).

Si l'on s'intéresse aux formes stabilisées d'interaction sociale dans les organisations (réunion, " conventions ", " entretiens annuels d'évaluation ", " coaching ", voire " négociations ", etc.), on ne peut quêtre surpris et en désaccord avec l'usage du terme "conversation» pour tous ces moments et encore, en entreprise, des «séminaires» (ce sont les moments qui font une grande partie du terrain de Lambotte et Wathelet). Certes les salariés « contribuent » en trouvant une place dans les dispositifs communicationnels. Mais dans le cours d'activité et « in situ ", il me semble notable, pour l'ethnolinguiste, que les situations de communication sont cadrées par des espaces dédiés, une organisation puissante dans le cadrage expressif ${ }^{17}$. On est loin d'une symétrie généralisée des règles d'échange. Dès lors, " conversation ", comme méta-concept, met à mal l'analyse des rapports d'interactions au cours d'activité et dans les rapports distants de hiérarchie. Cela n'aide pas non plus à comprendre les jeux d'inclusion ou d'exclusion nécessaires d'acteurs dans les arènes communicationnelles ${ }^{18}$. Ce méta-concept ne favorise pas vraiment l'analyse des échanges qui contribuent au travail d'organisation, ou, pour reprendre l'analyse de Strauss, au travail d'articulation - perspectives certes différentes des approches des "formes organisationnelles » ou des analyses des transformations des « modèles d'organisation ou de management »-. Ma perspective est centrée les «formes communicationnelles » et les échanges lors des activités et du travail ; or on ne peut interpréter « les communications au travail » comme développant simplement des formes de coordination et de coopération. Il serait dommageable de mettre de côté la violence des rapports sociaux dans l'entreprise ou, plus simplement la force des cadres symboliques des communications, ou encore les pouvoirs de décision de (dés)organisation et d'encadrement de l'action qui marquent les échanges en contexte de travail (qu'il s'agisse d'activités de production, de service, d'administrationgestion). Pour avancer sur ces points, il est important de développer - en sus de lobservation des inventions de formes et des petites organisations personnelles qui permettent de mémoriser et d'échanger pour faire du «bon boulot " - une méthodologie d'étude des cadres, des équipements et des normes communicationnels.

17 On trouve dans La dispersion au travail de C.Datchary (Datchary 2011 : 160) avec son étude du régime d'engagement de type "familiarité ", au travail, que celui-ci fait construire des conventions collectives locales ; plus les personnes sont familières plus elles peuvent ignorer les contraintes rituelles qui président à l'organisation des conversations que Goffman avait étudiée (Goffman 1987).

18 Cette difficulté conceptuelle étant posée, je peux reconnaître que depuis une dizaine d'années les chercheurs se référant à la CCO ont développé de nombreuses réflexions sur l'autorité, l'auctorialité (" authoring ») et les effets de pouvoir qui y sont associés. 


\section{Des approches qui peuvent s'articuler?}

Je ne voudrais pas qu'on se trompe : je ne fais pas une critique «interne" et j’ai besoin du travail et des avancées de la CCO. J'avance plusieurs idées.

Disons d'abord que je pense que la discussion actuelle se fait entre chercheurs dont les méthodes se confrontent par l'échange, mais dont la focale est un peu différente. Des chercheurs veulent développer une théorie de l'organisation (CCO : organizing par la com), d'autres une théorie du management et de l'activité (altération des formes organisationnelles, forces sociales et dispositifs de gouvernement, dispositifs de finalité-prescriptifs, de procédés-productifs, d'enrôlement-de contrôle), d'autres une théorie des communications en contexte de travail (formes communicationnelles, normes d'expression au travail, équipements et contribution à l'activité par les échanges et les productions langagières). Identifier ces différences permet de comprendre les enjeux des développements théoriques de chacun, mais aussi les enjeux d'invention de méthodes.

Or je pense que l'approche interactionniste des organisations et son évolution peuvent permettre de mieux documenter des phénomènes "vifs " pour les chercheurs en " communication organisationnelle ». Ainsi, et pour ne donner qu'un exemple, je pense que le travail de Fr. Cooren permet de remettre en chantier des questions politiques dont l'analyse s'était restreinte à l'économie des échanges linguistiques de Bourdieu : je pense en particulier aux analyses de l'époque concernant les "porte-parole», à l'occasion de la discussion critique que Bourdieu faisait des thèses d'Austin. Cette question est nécessairement revisitée dans une perspective de sociologie de la traduction pour qui nombre d'actants sont des hybrides « objets-machines-langage » : "porte-parole » chez Callon-Latour a un autre sens.

Le travail de Fr.Cooren me semble permettre de documenter les questions suivantes: qui assure un travail ordinaire de présentification d'aspects définitoires de l'organisation ? Et quand? Qui assure, par le rendu compte, la " reddition de compte », le reporting (" account », Latour 2006), le maintien du sens et d'objectifs pour partie identitaires ? Et quand ? Qui met en ordre et -élément de cette mise en ordre-hiérarchise l'agenda communicationnel qui permet de réaliser l'action collective ? Et quand ? Bref, comment des salariés « s'autorisent» de l'organisation.

Or c'est l'analyse des interactions, coûteuse, chronophage pour nous chercheurs, qui me semble la mieux à même de montrer comment se fait ce travail (portage de parole, réinterprétation de textes) et quels en sont les actants.

Reste que, pour moi, la critique majeure de la $\mathrm{CCO}$ que je développe dans ce texte est que définir une approche communicationnelle par le texte et la conversation, voire les textes et les interactions est insuffisant pour développer une théorie des échanges (par des textes et des interactions) en contexte de travail. Pour ma part, je propose qu'une théorie de la communication en 
contexte de travail soit attentive à ce que la communication " adressée » est une action qui tente le déplacement de l'autre, concept qui me paraît central. La construction de l'autre comme destinataire (dans l'énoncé et l'énonciation donc), la " disposition de l'autre ", dans le cadre matériel et symbolique où s'installe l'échange, notamment, sont des tentatives langagières pour déplacer l'autre ou les autres que l'on cherche à " prendre » dans l'échange comme interlocuteur, partenaire ou public. Un avantage de cette définition est d'envisager dans les échanges (les interactions) le travail communicationnel de chacun, et leurs contributions à l'activité et aux opérations sociales combinées qui font l'activité. Un autre est qu'il permet de comprendre en amont les enjeux économiques du déplacement matériel et symbolique de l'autre, de mobilisation économique et juridique pour installer les cadres des échanges, leurs équipements, et encore des actions comme l'invention de dispositifs qui visent à cadrer durablement le management de l'activité et le travail des salariés.

\section{BIBLIOGRAPHIE}

BACHMAN Ch.,LINDENFELD J., SIMONIN J., 1981, Langageet communications sociales, Hatier-Crédif.

BAZET I. \& MAYÈRE A., 2016, "Écrire dans et sur le dossier patient : disciplinarisation équipée de l'écrire et discipline de la communication », in Delcambre P. \& Matuszak, C., 2016, Ecrire au magistrat. Nouvelles normes, nouvelles contraintes. Presses du Septentrion, p. 143-167.

BOURDIEU P., 1982, Ce que parler veut dire. L'économie des échanges linguistiques, Fayard.

CALLON M., 2006, "Sociologie de l'acteur réseau », in Akrich M., Callon M., Latour B., 2006 Sociologie de la traduction. Textes fondateurs, Mines Paris, p. 267-276.

CHÉRONNET H., 2016, « La Protection judiciaire de la jeunesse à l'épreuve de la nouvelle gestion publique. Remaniement des légitimités, concurrence des normes et déstabilisation des identités professionnelles ", in Delcambre P. \& Matuszak C., 2016, Écrire au magistrat. Nouvelles normes, nouvelles contraintes. Presses du Septentrion, p. 2342.

COOREN Fr., 2010, «Comment les textes écrivent l'organisation. Figures, ventriloquie et incarnation ", Etudes de communication, n 34, p. 23-40.

COOREN F., 2013, Manières de faire parler: Interaction et ventriloquie. Bord de l'eau.

COOREN Fr., 2016, Le texte selon l'approche CCO: Agentivité, itérabilitée et ventriloquie, présenté aux journées Orgछ $C_{\text {Co, Mons }}$ 29/01/2016.

DATCHARY C., 2011, La dispersion au travail, Octarès Éditions.

LA BROISE P. de, \& MATUSZAK C., 2015, Lécrit professionnel en contexte de transformation politico-juridique et managériale. Normes, professionnalité et organisation. Maison Européenne des SHS Lille Nord de France. 
DELCAMBRE P., 1997, Écriture et communications de travail. Pratiques d'écriture des éducateurs spécialisés, Presses Universitaires du Septentrion.

DELCAMBRE P., 2010, «Written and Oral Communication in the WorkplaceDeployment, Stabilized Forms of Interactions, and Workload: An Organizational Approach », Management Communication Quaterly, 2010, 24(4) 635-642.

DELCAMBRE P., 2016a, "Cadrages et prescriptions : des effets de contrainte sur l'activité... et sur l'activité d'écriture aux magistrats ? ", in Delcambre P. \& Matuszak C., 2016, Écrire au magistrat. Nouvelles normes, nouvelles contraintes. Presses du Septentrion, p. 105-141.

DENIS J., 2007, « La prescription ordinaire. Circulation et énonciation des règles au travail », Sociologie du travail, 49 (4), p. 496-513.

DESHAYES F. \& LAÉ J-Fr., 2016, « Quand les usagers écrivent et constituent leurs dossiers : agir le droit pas à pas ", in Delcambre P. \& Matuszak C., 2016, Écrire au magistrat. Nouvelles normes, nouvelles contraintes. Presses du Septentrion, p. 303-318.

DUJARIER M-A., 2006, "La division sociale du travail d'organisation dans les services ", Erès/Nouvelle revue de psychosociologie, 2006/1, p. 129-136.

DUJARIER M-A., 2015, Le management désincarné. Enquête sur les nouveaux cadres du travail, La Découverte.

FORNEL M. de \& LÉON J., 2000, « L'analyse de conversation, de l'ethnométhodologie à la linguistique interactionnelle », HEL (Histoire Epistémologie Langage), Volume $22 \mathrm{n}^{\circ} 1$, p. 131-155.

FRAENKEL B., 2006, "Actes écrits, actes oraux : la performativité à l'épreuve de l'écriture ", Études de communication, n 29, p. 69-93.

GARDIN B., 1976, « Discours syndical, discours patronal », Langages, 1976, n 41, «Typologie du discours politique », p. 13-46.

GOODY J., 1986, « L'État, le bureau et le dossier », in La logique de l'écriture, Armand Colin, p. 97-131.

HELLER Th., 2016, « De l'accélération dans la justice des mineurs », in Delcambre P. \& Matuszak C., 2016, Écrire au magistrat. Nouvelles normes, nouvelles contraintes, p. 43-78.

HÉMONT Fl., 2011, Une approche communicationnelle du «développement fournisseur ». Le cas des rapports clients-fournisseurs dans l'aéronautique, Thèse Université de Toulouse décembre 2011 (Dir : Grabot et Mayère).

JOJCZYK J., LAMBOTTE Fr., MNASRI S., WATHELET E., 2016, « Les articulations des textes et des conversations dans l'approche de la communication constitutive des organisations ", Revue française des sciences de l'information et de la communication mis en ligne le 01 septembre 2016, http://rfsic.revues.Org/2197; 10.4000/ rfsic. 2197

JOSEPH I., 1998, Erwing Goffman et la microsociologie, PUF.

HELLER Th., 2016, «De l'accélération dans la justice des mineurs », in Delcambre P., \& Matuszak C., 2016, Écrire au magistrat. Nouvelles normes, nouvelles contraintes. Presses Universitaires du Septentrion, p. 43-78. 
JURMAND J-P., 2009, « La professionnalité éducative à l'épreuve de son historicité », Société et jeunesse en difficulté, n 7, Printemps 2009, URL://sjed.revues.org/index6109. html

LA BROISE P. de, \& MATUSZAK C., 2015, Lécrit professionnel en contexte de transformation politico-juridique et managériale. Normes, professionnalité et organisation. Maison Européenne des SHS Lille Nord de France.

LAMBOTTE Fr. \& WATHELET E., 2016 - à paraître - « Le cycle texteconversation comme dispositif d'accompagnement du changement "catalyseur" des tensions au sein d'une organisation publique en restructuration ", Revue de communication E Management (3)

Langages, 1981, $\mathrm{n}^{\circ}$ 62, "Analyse du discours politique ».

LATOUR B., 2006, Changer de société, refaire de la sociologie, La Découverte.

LE MOËNNE Ch. \& GALLOT S. 2015, « Les recherches en communication organisationnelle en France : quelques éléments de bilans et de perspectives. Entretien avec Christian Le Moënne, réalisé par Sidonie Gallot », Communiquer, revue de communication sociale et publique, 13/2015 « Perspectives en communication $1^{\text {re }}$ partie », p. 123-143.

Le Moënne, Ch., 2015, " Pour une approche "propensionniste" des phénomènes d'information-communication organisationnelle. Émergence et différenciation des formes sociales », Communication et organisation, 47/2015.

MOISDON J.-C., dir., 1997, Du mode d'existence des outils de gestion, Seli Asran.

RICEUUR P., 1986, Du texte à l'action : essai d'herméneutique, II. Paris: Seuil.

ROSA H., 2013, Accélération, une critique sociale du temps, La Découverte.

SALLÉE N., 2010, " Les éducateurs de la Protection judiciaire de la jeunesse à l'épreuve de l'évolution du traitement pénal des jeunes délinquants ", Champ pénal, https://champpenal.revues.org//7756

TAYLOR J. R., COOREN F., GIROUX N. \& ROBICHAUD D., 1996, The communicational basis of organization: Between the conversation and the text. Communication Theory, 6(1), 1-39.

TAYLOR J. R. \& EVERY E. J.van, 2000, The Emergent Organization. Communication as Site and Surface, Lawrence Erlbaum Associates.

TAYLOR J., GROLEAU C., HEATON L., EMERY E. van, 2001, The computerization of work. A communication perspective, Sage.

TAYLOR J. R. \& EVERY E. J. van, 2011, The situated organization: Case studies in the pragmatics of communication, Routledge.

TAYLOR J. R. \& EVERY E. J. van, 2014, When organization fails: Why authority matters, Routledge.

TERSSAC G. de., 2002, (dir) Le travail : une activité collective, Octarès.

VASQUEZ C. \& COOREN Fr., 2013, «Spacing Practices : The Communicative Configuration of Organizing Through Space, Time, Communication theory, 23 (1), p. 25 47.

VION R.,1992, La communication verbale. Analyse des interactions, Hachette Supérieur.

WINKIN Y., 2001, La nouvelle communication. Textes de Bateson, Birdwhistell, Goffman, Hall, Jackson, Scheflen, Sigman, Watzlawick recueillis et présentés par Yves Winkin, Seuil. 
Résumé : En prenant comme terrain une direction ministérielle d'un ministère en France cet article repose sur une observation de longue durée du travail d'organisation consécutif à de nouvelles prescriptions; il s'intéresse tout particulièrement aux échanges et au travail textuel que ces prescriptions induisent. Mettre en place et contrôler la mise en œuvre d'une prescription suppose des échanges nombreux et un travail textuel support du travail d'organisation, mais aussi des audits ; les dispositifs d'informatique de gestion visent aussi à évaluer l'installation du prescrit; enfin des référentiels légitiment la connaissance qu'a l'organisation d'une activité procéduralisable sur laquelle s'appuie la prescription. Cette étude est l'occasion d'interroger ici les concepts de « texte » et de « conversation » tels que la $\mathrm{CCO}$ les a développés. Dans une perspective d'analyse des " formes communicationnelles ", l'auteur préfère envisager la multiplicité documentaire des textes et se refuse à considérer comme " conversation " la plupart des échanges structurés par les formes et les espaces de communication.

Mots-clés : travail d'organisation, prescription, texte, échanges au travail, conversation.

\begin{abstract}
This article is based on long-term field observations of the organizational work resulting from new managerial prescriptions emanating from French ministerial policy. We demonstrate the importance of oral exchanges, textual production and service audits during the period of implementation of these prescriptions. In addition, implementation and evaluation of prescriptions entail the use of specific computer management software. Finally, stabilized guidelines give legitimacy to prescriptions, and demonstrate the precision with which the organization's activity process is analyzed. This study allows us to question the CCO's concepts of «text " and "conversation ". In order to describe «communicational forms " available in workplaces, which provide the imposed framework for workers' exchanges and activities, we need to think about the roles and strengths of different texts, and to define the moments and forms of speech and transactions without defining these as «conversations".
\end{abstract}

Keywords: organizational work, managerial prescriptions, text, workplace exchanges, conversation. 